\title{
Resiliencia, autoestima y personalidad resistente en niños y adolescentes con antecedente de maltrato
}

Resiliency, self-esteem and hardiness in children and adolescents with history of abuse

\author{
Norma Ivonne González-Arratia López Fuentes* \\ Marisol Reyes Fonseca \\ José Luis Valdez Medina \\ Sergio González Escobar
}

\section{Resumen}

El objetivo de este estudio es indagar la relación entre las variables resiliencia, autoestima y personalidad resistente en niños con antecedente de maltrato que se encuentran actualmente en una institución del sistema integral de la familia (DIF) de la ciudad de Toluca, Estado de México. La muestra se compone de 75 niños y adolescentes entre 10 y 18 años de edad con antecedente de maltrato físico, abuso sexual, negligencia y combinación de estos tipos de maltrato. Los datos indican que no existe diferencia estadísticamente significativa en las variables respecto al tipo de maltrato, pero sí existe correlación positiva moderada entre las variables (resiliencia y autoestima y personalidad resistente). Se concluye que es necesario profundizar en el estudio de la resiliencia y su asociación con autoestima y personalidad resistente, así como plantear la necesidad de que la institución provea las condiciones necesarias para el desarrollo y fortalecimiento de estas características de personalidad de los niños y adolescentes, a fin de que influya positivamente sobre la capacidad de enfrentarse a situaciones y salir fortalecidos, es decir, ser resilientes.

\section{Abstract}

The aim of this study was to investigate the relationship between resilience, self-esteem and hardiness in children with a history of abuse that are currently in an institution of the Mexico's Integral Family System (DIF) at the city of Toluca. The sample comprised 75 children and adolescents between 10 and 18 years old with a history of physical abuse, sexual abuse, neglect and combinations of these types of abuse. The data indicate no statistically significant differences in the variables by type of abuse; yet, a moderate positive correlation between these variables (resiliency, self-esteem and hardiness) was found. It is concluded that further studies on resilience and its association with self-esteem and hardiness are needed; also that the institution is expected to provide the necessary conditions for the development and strengthening of these personality characteristics of children and adolescents, since they positively influence the ability to cope with situations, and becoming stronger and resilient. 


\section{Introducción}

En el mundo actual la resiliencia es más importante que nunca, dado que los individuos que poseen más habilidades para resistir tienen una ventaja significativa frente a quienes se sienten desamparados o quienes reaccionan como víctimas. $Y$ es precisamente la investigación en psicología que ha llevado a identificar y a conocer qué es lo que distingue a las personas que son más resilientes que otras.

En México, para 1999 se reportaron un total de 12,516 niños y 12,433 niñas maltratados. De estas cifras el estado con mayor índice fue Coahuila $(4,150)$, seguido de Nuevo León $(3,067)$ y en tercer lugar el Estado de México $(1,885)$. El tipo de maltrato más frecuente en el Distrito Federal fue el físico con $44 \%$, en Coahuila la omisión de cuidados con $35 \%$, en Sinaloa con un $72 \%$ al igual que Nuevo León con un $58 \%$. Sin embargo, estas cifras pueden no ser concluyentes, ya que en muchas ocasiones no es denunciado.

En el caso del Estado de México, lugar donde se hace la investigación, actualmente las políticas asistenciales son operadas por el sistema Integral de la Familia del Estado de México (DIFEM), y están dirigidas a la protección de poblaciones vulnerables, particularmente niños en situación de desamparo, madres, ancianos y discapacitados, a través de apoyo como leche para los niños, despensas, ropa, medicinas, sillas de ruedas, albergues y casa cuna (Guadarrama, 2001). Y las funciones de los servicios asistenciales (Villa Hogar, Villa juvenil, Albergue Temporal Infantil), son el proporcionar casa-habitación, alimento y vestido a cada joven ingresado, proporcionar el ingreso a una institución educativa de acuerdo al nivel y grado académico que le corresponda para continuar su desarrollo, ofrecer diferentes opciones de capacitación técnica como artesanal para un trabajo productivo y brindar atención médica y psicológica.

La investigación en resiliencia se ha enfocado en los niños y adolescentes en situaciones de riesgo asociadas con variables como la pobreza, marginalidad, maltrato y vínculos familiares. Los resultados a nivel general demuestran que los niños que están en mayor situación de riesgo son aquellos que se ven enfrentados a una acumulación de circunstancias adversas tales como: dificultades económicas, situación de pobreza, enfermedad mental de alguno de los padres, prácticas de crianza inconducentes a su desarrollo o bien abuso y conflictos familiares (Vinaccia, Quiceno y Moreno, 2007).

La experiencia de abuso tiene varias respuestas. Algunos individuos tienen severos trastornos de personalidad, mientras que otros no. Se cree que las diferencias en la respuesta al abuso se deben a la presencia de un factor protector en los niños conceptualizado como resiliencia (Browne y Finkelhor, 1986).

En la investigación tradicional se ha manejado la idea de que un niño maltratado se convertirá con toda probabilidad en un adulto maltratador. Sin embargo, esta afirmación no es así, puesto que se ha observado que no necesariamente un niño herido está condenado a ser un adulto fracasado, como lo refiere Tomkiewiz (2001) ya que estos niños salen adelante en dos de cada tres casos en los que no hay atención especial y mucho más a menudo si se ayuda al niño correctamente (Williams, 2001; lonescu, 1992, en Manciaux, 2003). Asimismo es muy popular la idea de que también los adultos maltratadores fueron maltratados en su infancia (Aracena, Castillo, Cumsille, Bustos y Román, 2000). Al respecto es poco claro la relación entre la historia de abuso y maltrato actual, además, los estudios se basan en el reporte de observadores que conocen la condición de maltratador o no maltratador de los sujetos estudiados (Kaufman y Zigler en Ammerman y Versen, 1990).

A partir de la aplicación del concepto resiliencia a la fecha no existe una definición universalmente aceptada y casi todas las que figuran en la bibliografía intentan explicarla en términos generales como: proceso, capacidad, crecimiento, enfrentamiento y habilidad. Pero existe 
un consenso general, de que la resiliencia es un proceso que refleja una relativa adaptación positiva a pesar de contextos de riesgo, adversidad significativa o trauma y como aquella cualidad de las personas para resistir y rehacerse ante situaciones traumáticas o de pérdida.

Se considera que la resiliencia es la aptitud para soportar las crisis, estrés y adversidades en forma positiva recobrando la fortaleza o resistencia para salir airosos de la situación. Implica el enfrentar los problemas que producen estrés advirtiendo posibilidades de superarlos y sobre todo, de recuperarse y fortalecerse con la experiencia. Desde esta concepción, entonces, los problemas y situaciones constantes de crisis pueden ser enfrentados y superados buscando las particularidades positivas que llevan a mejorar la situación futura. Por tanto, la capacidad de resiliencia consiste en recuperarse de los conflictos no únicamente dejando que sólo desaparezca la crisis, sino, impulsando cambios y fortaleciendo las defensas ante la reaparición de los mismos.

Es así que con esta visión más amplia se está de acuerdo con la definición de Suárez Ojeda (1997), en que la resiliencia significa una combinación de factores que permiten a un niño, a un ser humano, afrontar y superar los problemas y adversidades de la vida, y construir sobre ellos.

Por lo que la resiliencia será entendida en esta investigación como: el resultado de la combinación y/o interacción entre los atributos de individuo (internos) y su ambiente familiar, social y cultural (externos) que lo posibilitan para superar el riesgo y la adversidad de forma constructiva (González Arratia, 2007).

Respecto a los intentos por desentrañar los posibles correlatos del constructo resiliencia con otras variables psicológicas, la autoestima y personalidad resistente resultan relevantes de analizar, con el fin de obtener mayor información en relación a la caracterización de la resiliencia en niños y adolescentes con antecedente de maltrato y que a continuación se revisan.

\section{Autoestima}

La autoestima ocupa un lugar importante dado que los resultados de investigación sugieren que éste es un factor predictor de la resiliencia; estos hallazgos son consistentes en considerar que existe estrecha relación entre resiliencia y autoestima (Davey Goettler y Walters, 2003; Dumont y Provost, 1999, González Arratia, 2007).

Coopersmith (1967), definió la autoestima como la evaluación que el individuo hace y mantiene en relación a sí mismo; expresa una actitud de aprobación o de rechazo e indica el grado en el cual el individuo se siente capaz, significativo, exitoso y valioso. Desde la misma perspectiva de las actitudes se encuentra Rosenberg y, que en este estudio se considera que la autoestima es una actitud positiva o negativa, de aprobación o desaprobación respecto de sí mismo (Rosenberg, 1965).

El estudio de la autoestima y resiliencia se hace importante, ya que como lo explica Löesel (1989), entre los recursos más importantes con los que cuentan los niños resilientes, se encuentra el haber vivido experiencias de autoeficacia, autoconfianza y contar con una autoimagen positiva, es decir con una autoestima positiva.

La autoestima determina el grado en que es capaz y valioso; entre mejor actitud tenga una persona hacia sí mismo, se podrá enfrentar mejor al mundo, aprovechando más y en todo su potencial las habilidades con las que cuente. En el caso de los niños, una mejor disposición hacia sí mismos, mejorará su adaptación al medio ambiente y también su capacidad para resistir presiones y enfrentar las situaciones que se le presenten. Así mismo, se ha observado que una persona que se siente querida, valiosa, capaz, se acepta a sí misma; es confiado, gusta de la proximidad afectiva, entre otras cosas. Puede adquirir e ir utilizando habilidades y estrategias para enfrentar situaciones difíciles. Además, la autoestima determina la forma cómo el niño (a) se va sobreponiendo y probando en el momen- 
to de enfrentar las dificultades y las crisis (Lara, Martínez y Pandolfi, 2000).

Además Rutter, (1985, en Walsh, 2004) apuntó que una alta autoestima y una buena dosis de eficacia personal, tornaban más probable el éxito en la superación de las dificultades; en tanto que la baja autoestima aumenta la posibilidad de que cada acontencimiento adverso origine otro.

Entre los estudios sobre resiliencia y autoestima es interesante destacar el trabajo clásico de Werner, (1989), ya que, concluye que estos niños fueron creciendo con un alto nivel de autoestima al poder contar con alguien incondicional y al mismo tiempo sabiendo que sus esfuerzos eran reconocidos y fomentados. De esta forma, sostiene que todos los niños que han padecido algún sufrimiento, pero poseen autoestima alta, tuvieron la influencia de por lo menos una relación cariñosa y estrecha con un adulto significativo (Miguens, 2004).

La autoestima está relacionada con altos niveles de bienestar a pesar de los eventos adversos. Es un recurso (variable moderadora) para el afrontamiento a las amenazas y al estrés. Además, poseer una adecuada autoestima es una precondición positiva para movilizar o simplemente aceptar el apoyo social, y así ser una variable mediadora para el manejo de problemas (Moreno, Alonso y Álvarez, 1997; Jiménez, 2008).

Una de las consecuencias de tipo emocional ante la vivencia del maltrato según Loredo (1994), es precisamente la autoestima, ya que los niños que han sido víctimas de maltrato consideran que sus sentimientos y deseos internos no tienen importancia; e incluso consideran que no eran importantes para sus padres. Se perciben inadecuados en su cuerpo y en su inteligencia, se aprecian torpes e inseguros de quién y qué clase de persona son y con esto se muestra cierta incapacidad para enfrentarse y dominar las situaciones críticas que se le presentan en su vida cotidiana.
Este mismo autor, refiere que además los niños maltratados crecen con esa constante necesidad de ser queridos, de sentirse parte de un ambiente, de ser aceptados por un grupo y de poder expresar sus sentimientos de enojo y agresión y no encuentran la manera de hacerlo, terminando en el refugio de la soledad. Desde pequeños viven con el agobio de sentirse desaprobados, buscando cualquier aprobación de las personas que lo rodean. En la etapa escolar se hallan temerosos y en alerta para encontrar la aceptación de los maestros y demás compañeros, pero también para detectar cualquier signo de desaprobación y de crítica (Loredo, 1994).

\section{Personalidad Resistente}

Una variable más incluida en este estudio, es la personalidad resistente y es la castellanización del concepto proveniente de la lengua inglesa hardiness cuya traducción significa dureza, solidez, severidad y su propio significado al referirse a una persona dura y fuerte. Según sus principales autores (Kobasa, Maddi y Kahn, 1993), el concepto de personalidad resistente se desarrolla a través del estudio de aquellas personas que ante los hechos vitales negativos parecen tener características de personalidad que les protegen. Además, se asocia con una tendencia a percibir los potenciales eventos traumáticos en términos menos amenazadores y sus efectos están mediados por mecanismos de evaluación del ambiente y de afrontamiento (Kobasa, 1979).

La personalidad resistente comparte con la resiliencia el atributo que presupone un riesgo pero con un grupo específico de rasgos de los individuos, más que a la combinación de riesgos, competencias y factores o recursos de protección, como ocurre en la resiliencia (Jiménez, 2008).

Desde esta perspectiva, Kobasa (1979) desarrolló el concepto de personalidad resistente, la cual se caracteriza por un fuerte sentimiento 
de control sobre la vida y de compromiso con ciertas metas y objetivos, que fungen como cualidades que interactúan constantemente $\mathrm{e}$ inciden en la actitud, estado de ánimo, comportamiento y toma de decisiones relacionadas con algunas estrategias de afrontamiento.

La personalidad resistente se asocia con la resiliencia, la buena salud y el buen funcionamiento en condiciones de estrés (Bartone, 1999, Kobasa, 1979, Maddi y Kobasa, 1984).

Una investigación como la de Feinaver, Hiltol y Callahan (2003), explora los efectos negativos del abuso sexual en una muestra de adultos sobrevivientes al mismo y refieren que la personalidad resistente puede ser un factor importante para ayudarlos.

Kobasa propone en su modelo la importancia que tiene una concepción propositiva en el estudio de la personalidad. Esta perspectiva permite considerarla básicamente como una realidad dinámica que establece una relación con la situación específica de cada momento a partir del cambio y la interacción y no solamente la reacción pasiva a los estresores (Moreno, Alonso y Álvarez, 1997).

Por ello, propone un conjunto de variables que capacitan al sujeto para enfrentar activamente al estrés y en el que están implicados tres componentes, los cuales se consideran claves en el estudio de la personalidad resistente.

1. Compromiso. Las personas con compromiso poseen tanto las habilidades como el deseo de enfrentarse exitosamente a situaciones de ansiedad, la cual ayuda a mitigar la amenaza percibida de cualquier estímulo estresante en un área específica de la vida. Presupone la autenticidad, el reconocerse a sí mismo como persona valiosa y capaz de lograr determinadas metas y propósitos existenciales que se proponga, el ser capaz de tomar responsablemente decisiones congruentes con los principios y valores y, como consecuencia, comprometerse con las consecuencias de las mismas.
2. Control. Las personas con control buscan explicaciones sobre el por qué de los acontecimientos tanto en las acciones de los demás como en su propia responsabilidad. Permite al individuo percibir muchas de las situaciones estresantes, las consecuencias predecibles debidas a su propia actividad, manejar los estímulos en su propio beneficio siendo capaces de interpretar los sucesos estresantes e incorporarlos dentro de un plan de metas transformándolo en algo consistente con el sistema de valores del organismo. Es decir, el sujeto está convencido de poder intervenir en el curso de las situaciones.

3. Reto-desafío. Es la medida en que el sujeto percibe que las situaciones complicadas son una oportunidad de crecer y considera los cambios como favorables en lugar de percibirlos como algo perjudicial para el individuo (Kobasa, 1982).

Diferentes estudios muestran que la personalidad resistente está relacionada con la salud; asimismo, se ha encontrado que las personas con altas puntuaciones en personalidad resistente afrontan de modo adecuado el estrés, la enfermedad y las condiciones adversas de la vida, además suelen promover estilos de vida saludables (Kobasa, Maddi y Kahn, 1993).

Existe evidencia de que la personalidad resistente, se relaciona positivamente con la autoestima, asertividad y extraversión y, negativamente con depresión y ansiedad, además de que predice la satisfacción con el trabajo (Ghorbani, 2005).

\section{Maltrato infantil}

El síndrome del niño maltratado es según la Organización Mundial de la Salud, toda forma de abuso físico o mental, descuido o trato negligente, malos tratos o explotación incluido el abuso sexual a niños por parte de los padres, representantes legales o cualquier otra persona que lo tenga a su cargo. 
También se le considera como cualquier acción u omisión realizada por individuos, instituciones o por la sociedad en su conjunto y todos los estados derivados de estos actos o de su ausencia que priven a los niños de su libertad o de sus derechos correspondientes y/o que dificulten su óptimo desarrollo (Frías, Castell e Ibarra, 2000). La definición de maltrato encierra dos tipos de actos: hacer o dejar de hacer cosas. Por otro lado, el ejercicio de la violencia identificada como maltrato tiene lugar, sobre todo, en el seno de la familia (Reppucci, Britner y Woolard, 1997).

La prevención del maltrato infantil es un tema que preocupa a las instituciones de gobierno porque los menores que son expuestos a la violencia están en grave riesgo de desarrollar desórdenes psicológicos y problemas sociales como la delincuencia (Widom, 1989). Además, particularmente el abuso sexual infantil incrementa en las víctimas la depresión, ansiedad e incluso en algunos individuos pueden presentarse severos disturbios de personalidad, mientras que en otros no. Investigaciones clínicas revelan que estas diferencias se deben a un factor protector denominado resiliencia (Browne y Finkelher, 1986).

Por otro lado, si bien muchos estudios han encontrado relaciones positivas entre resiliencia, personalidad resistente y autoestima aún queda mucho por conocer respecto a su relación entre estas variables. De tal forma que la información concerniente a la naturaleza de la relación entre estas variables se hace necesaria especialmente en nuestro contexto sociocultural.

Por lo tanto, en esta investigación se tienen dos objetivos. En primer lugar, examinar la relación entre las variables resiliencia, autoestima y personalidad resistente en niños y adolescentes que tienen antecedentes de maltrato y que actualmente se encuentran en una institución a fin de constatar si estas variables están asociadas entre sí y de qué manera y, como un segundo objetivo es observar si se presentan valores di- ferenciados en función del antecedente de maltrato.

\section{Método}

\section{Participantes}

La muestra es no probabilística de tipo intencional, conformada por un total de 75 niños y adolescentes de ambos sexos (24 mujeres, 51 hombres) entre 10 y 18 años de edad, que se encuentran actualmente en una institución perteneciente al Sistema para el Desarrollo Integral de la Familia (DIF), cuya finalidad es la atención a los menores en situación extraordinaria y cuentan con diferentes albergues: el temporal infantil, villa hogar y villa juvenil. El motivo de ingreso de los menores fue por el hecho de que fueron víctimas de maltrato $\mathrm{y}$ constatado por la institución (médico y judicial) y por la historia clínica según lo reportado por el Departamento de Psicología de la misma institución como: maltrato físico (12), abandono o negligencia (17), abuso sexual (3), combinación de maltrato (43). El escenario del maltrato y abuso había sido en sus hogares ocasionado por mujeres maltratadoras de sus hijos.

\section{Instrumentos}

1. La resiliencia fue medida con el Cuestionario de Resiliencia (González Arratia, 2011). Es un instrumento de autoinforme desarrollado en México, para niños y adolescentes que mide factores específicos de la resiliencia, consta de 32 reactivos con un formato de respuesta tipo Likert de cinco puntos (el valor 1 indica nunca y el 5 siempre). Del análisis factorial exploratorio ortogonal (varimax) y con base al punto de inflexión del gráfico de sedimentación, expectativas teóricas e interpretabilidad, se optó por la solución de tres factores que explican una varianza acumulada de $37.82 \%$ y un coeficiente de confiabilidad Alpha de Cronbach con los 32 reactivos de 0.91 , dividida en tres dimensiones que son: 
a. Factores protectores internos: se refiere a funciones que se relacionan con habilidades para la solución de problemas (alpha $=.80$, con 14 reactivos).

b. Factores protectores externos. Se refiere a la posibilidad que considera el individuo que tiene de contar con apoyo de la familia y/o personas significativas para el individuo (Alpha= 0.73, con 11 reactivos).

c. Empatía. Denota comportamiento altruista y prosocial (Alpha $=0.78$ con 7 reactivos).

2. Cuestionario de Autoestima de Verduzco (2004) que consta de 32 reactivos con dos opciones de respuesta Si o No. La autora reporta el $52.3 \%$ de varianza y un Alpha de Cronbach total de .83. Se dividen en seis factores que son:

a. Devaluación a nivel social (alpha=0.75, con 9 reactivos).

b. Cumplir con expectativas sociales (alpha $=0.71$, con 3 reactivos).

c. Inseguridad (alpha $=0.62$, con 6 reactivos).

d. Familia (alpha $=0.52$, con 5 reactivos).

e Aspectos negativos de la autoestima (alpha $=.52$, con 6 reactivos).

f. Escuela (alpha=.59, con 3 reactivos).

Con el fin de facilitar la calificación en esta investigación se obtuvieron puntajes para cada una de las dimensiones y posteriormente se clasificaron en 4 niveles: autoestima alta, tendencia a alta autoestima, tendencia a baja autoestima y baja autoestima para la interpretación del mismo. Para esta investigación se obtuvo un coeficiente de confiabilidad Alpha de Cronbach satisfactorio (.84).
3. Cuestionario de personalidad resistente se utilizó el Inventario de puntos de vista personales de Moreno, Alonso y Álvarez (1997). Consta de 21 reactivos y 4 opciones de respuesta que va de 1 (totalmente en desacuerdo), 2 (en desacuerdo), 3 (de acuerdo) al 4 (totalmente de acuerdo). En esta investigación se obtuvo una varianza de $32.87 \%$ y un Alpha de Cronbach total de .89. Sus dimensiones son:
a. Compromiso (alpha=0.59)
b. Control (alpha=0.62)
c. Reto / desafío (alpha $=0.62$ )

\section{Procedimiento}

Las aplicaciones se llevaron a cabo en el interior de cada albergue de la institución, formando pequeños grupos de menores de 4 a 5; los instrumentos se aplicaron en una sola sesión en un tiempo aproximado de 45 minutos, explicando dudas que surgieron al respecto, el motivo de la investigación y todos accedieron a participar de manera voluntaria. Los datos de tipo de maltrato fueron proporcionados por el departamento de Psicología de la misma institución, considerando aspecto éticos del trabajo con personas.

\section{Análisis de Datos}

Se obtuvieron las medias y desviación estándar para cada una de las dimensiones de resiliencia, autoestima y personalidad resistente y un análisis de varianza para observar diferencias según el antecedente de maltrato. También se llevó a cabo un análisis de correlación de Pearson, para estimar el grado de relación entre las variables considerando una $p \leq .05$. 


\section{Resultados}

Primero, se obtuvieron datos descriptivos como la media y desviación estándar de las dimensiones de cada uno de los instrumentos. En el caso de resiliencia, en la dimensión de factores internos, se obtuvo una media superior a la teórica y una menor en el caso de los factores protectores externos y empatía. En autoestima, las medias obtenidas indican una tendencia a baja autoestima y para personalidad resistente, los valores indican que la vulnerabilidad de la persona es alta (véase Tabla 1).

Al hacer un análisis de varianza de acuerdo a la variable antecedente del tipo de maltrato, no se encontraron diferencias estadísticamente significativas en ninguna de las dimensiones de resiliencia, autoestima y personalidad resistente. Por lo que a continuación se muestran los datos obtenidos de manera descriptiva por dimensión de cada uno de los constructos, específicamente media y desviación estándar, las cuales indican que en el caso de la resiliencia los niños y adolescentes, mostró una media más alta en las dimensiones de factores pro- tectores internos y externos a favor de los niños y adolescentes con antecedente de abuso sexual, mientras que existe la tendencia a mayor empatía en los que han tenido antecedente de maltrato físico.

En el caso de la autoestima, se encontró mayor devaluación, inseguridad, aspectos negativos y aspectos positivos de la familia en el caso de los niños con antecedente de abuso sexual; mientras que los niños y adolescentes con historia de negligencia indicaron mayor necesidad de cumplir con expectativas sociales, y para los que han sufrido una combinación de tipo de maltrato refieren más aspectos positivos en la escuela.

Para personalidad resistente, las medias obtenidas indican que los niños y adolescentes con antecedente de abuso sexual se encuentran con mayor compromiso y control, mientras que los que tienen antecedente de negligencia hay en mayor medida la dimensión de reto (véase tabla 2).

TABLA 1

Media y desviación estándar Resiliencia, Autoestima y Personalidad resistente.

\begin{tabular}{llcccc}
\hline & Media & D.E. & mínimo & máximo \\
\hline $1 \quad$ Factores protectores internos & 3.50 & .66 & 2.43 & 5.0 \\
& Factores protectores externos & 2.55 & .81 & 1.0 & 3.91 \\
& Empatía & 2.52 & .84 & 1.0 & 3.86 \\
2 Devaluación & 1.85 & .98 & 1.0 & 4.0 \\
& Cumplir con expectativas sociales & 1.82 & 1.14 & 1.0 & 4.0 \\
& Inseguridad & 2.16 & .93 & 1.0 & 4.0 \\
Familia & 2.84 & .54 & 2.0 & 4.0 \\
& Aspectos negativos de la autoestima & 2.20 & .86 & 1.0 & 4.0 \\
& Escuela & 1.97 & .80 & 1.0 & 4.0 \\
Compromiso & 2.96 & .63 & 1.0 & 4.0 \\
Control & 3.00 & .60 & 1.40 & 4.0 \\
Reto & 3.01 & .58 & 1.25 & 4.0 \\
\hline
\end{tabular}

1. Resiliencia, 2. Autoestima, 3. Personalidad resistente 


\section{Tabla 2}

Media y desviación estándar Resiliencia, Autoestima y Personalidad resistente según el antecedente de maltrato

\begin{tabular}{|c|c|c|c|c|c|c|c|c|}
\hline \multirow[b]{2}{*}{ Resiliencia } & \multicolumn{2}{|c|}{$\begin{array}{c}\text { Maltrato } \\
\text { físico }\end{array}$} & \multicolumn{2}{|c|}{ Negligencia } & \multicolumn{2}{|c|}{ Abuso sexual } & \multicolumn{2}{|c|}{$\begin{array}{c}\text { Combinación } \\
\text { de maltrato }\end{array}$} \\
\hline & Media & Ds & Media & Ds & Media & Ds & Media & Ds \\
\hline 1. Factores protectores internos & 3.86 & 69 & 3.76 & 68 & 4.19 & .90 & 3.68 & .63 \\
\hline 2. Factores protectores externos & 2.19 & .66 & 2.68 & .85 & 3.24 & 1.07 & 2.55 & .79 \\
\hline 3. Empatía & 2.76 & .82 & 2.63 & .89 & 2.14 & 1.22 & 2.43 & .81 \\
\hline \multicolumn{9}{|l|}{ Autoestima } \\
\hline 4. Devaluación & 1.50 & .52 & 1.76 & .97 & 2.33 & 1.15 & 1.95 & 1.06 \\
\hline 5. Cumplir con expectativas sociales & 1.75 & 1.13 & 2.17 & 1.28 & 1.66 & 1.15 & 1.72 & 1.09 \\
\hline 6. Inseguridad & 1.83 & .83 & 2.17 & 1.07 & 2.66 & 1.52 & 2.20 & .86 \\
\hline 7. Familia & 2.91 & .51 & 2.88 & .60 & 3.00 & .000 & 2.77 & .55 \\
\hline 8. Aspectos Negativos de autoestima & 2.16 & .83 & 2.17 & .95 & 2.33 & .57 & 2.30 & .88 \\
\hline 9. Escuela & 1.94 & .79 & 1.89 & .52 & 1.66 & .57 & 2.06 & .91 \\
\hline \multicolumn{9}{|l|}{ Personalidad Resistente } \\
\hline 10. Compromiso & 2.75 & .74 & 2.82 & .67 & 3.13 & .90 & 3.06 & .55 \\
\hline 11. Control & 2.80 & .67 & 3.04 & .65 & 3.18 & .75 & 3.18 & .55 \\
\hline 12. Reto & 2.95 & .62 & 3.08 & .64 & 2.50 & .43 & 3.02 & .54 \\
\hline
\end{tabular}

Como se puede ver en la Tabla 3, se indican los coeficientes de correlación de Pearson hallados entre las dimensiones de las escalas, en donde se encontraron correlaciones positivas moderadas y estadísticamente significativas que indican que a mayores factores protectores internos, mayor compromiso, a mayores factores protectores externos, mayores aspectos familiares (autoestima) y reto-desafío, y a mayor empatía, mayores necesidades de cumplir con expectativas sociales y familia (autoestima) (véase Tabla 3). 


\section{Tabla 3}

Correlación entre Resiliencia, Autoestima y Personalidad resistente

\begin{tabular}{|c|c|c|c|c|c|c|c|c|c|}
\hline & \multicolumn{6}{|c|}{ Autoestima } & \multicolumn{3}{|c|}{ Personalidad resistente } \\
\hline Resiliencia & 1 & 2 & 3 & 4 & 5 & 6 & 7 & 8 & 9 \\
\hline $\begin{array}{l}\text { Factores protectores } \\
\text { internos }\end{array}$ & & & & & & & $r=.356$ & & \\
\hline $\begin{array}{l}\text { Factores protectores } \\
\text { externos }\end{array}$ & & & & & $r=.368^{* *}$ & & & $r=.247^{*}$ & \\
\hline Empatía & & & & $r=.253^{*}$ & $r=.446^{\star *}$ & & & & \\
\hline
\end{tabular}

1.Autoestima: devaluación, 2. Inseguridad, 3. Cumplir con Expectativas Sociales, 4. Familia, 5. Aspectos Negativos de la autoestima, 6. Escuela, 7. Personalidad resistente: compromiso, 8.Control, 9. Reto. ${ }^{*} \mathrm{p}=.05,{ }^{\star \star} \mathrm{p}=.001$.

\section{Discusión}

Los resultados de este estudio indican que no se encontraron diferencias significativas en la variable antecedente respecto al tipo de maltrato. No obstante, los resultados muestran que las medias obtenidas sólo en la dimensión de factores protectores internos de la escala de resiliencia es superior a la media teórica; mientras que, en el caso de las dimensiones del cuestionario de autoestima como de personalidad resistente, éstas se encuentran por debajo de ésta. Lo cual indica una tendencia a presentar baja resiliencia, baja autoestima y una alta vulnerabilidad. Por lo que la hipótesis acerca de las diferencias de acuerdo al antecedente de maltrato no ha sido confirmada, lo cual requiere de mayor análisis.

En cuanto a la resiliencia, estos datos difieren a lo referido por Aracena, Castillo Cumsille, Bustos y Román (2000) en el sentido de que los participantes de este estudio no muestran características resilientes excepto en la dimensión de factores protectores internos. Asimismo, se ha podido observar que es constante en la investigación sobre maltrato físico encontrar una baja autoestima (Malinosky-Rummel y Hansen, 1993; Trianes y Gallardo, 1997), en los niños con estos antecedentes.

Esto tiene implicaciones importantes dado que hacen suponer que esta muestra de niños y adolescentes con antecedente de maltrato tiene un impacto importante en su personalidad. Por lo que parece relevante insistir en que es necesario proveer a estos niños y adolescentes de recursos psicológicos que les permitan salir adelante, así como en el cuidado, protección y educación que necesitan para llegar a la adolescencia e integrarse a la vida adulta de manera sana.

Así, ante la prevención de los malos tratos infantiles es necesario anclarse en la promoción de la salud. Y uno de los elementos está en la presencia de adultos significativos que pueden ejercer un impacto positivo para su desarrollo. Esto se debe a que en especial los niños por su condición dependen de los adultos, integrantes de sus familias; no obstante muchas veces son precisamente estos adultos los que alteran las condiciones de estabilidad cotidiana. Es entonces que ante la aparición de problemas y situaciones conflictivas, que afectan la permanencia del niño en su medio o bien cuando el medio natural no satisface los más elementales derechos y provoca reacciones adversas que pueden manifestarse en conductas conflictivas, es cuando se requiere de la intervención de una institución. Éstas son algunas razones, por las que los niños se encuentran en organizaciones, albergues, casa de asistencia y adopción (Loredo, 1994, Martínez, 2006).

Las funciones primordiales de estos centros de ayuda a niños abandonados son básicamente, las de cultivar la personalidad del menor y suplir en lo posible la carencia de hogar. La 
protección al niño huérfano o niño abandonado ha sido una de las formas clásicas de beneficiencia en todo tiempo; en la actualidad admite el régimen de internado como lo es el caso de los participantes de este estudio. Por lo que es necesario considerar que la institución en la que ahora se encuentran, puede actuar como un factor protector para estos pequeños y analizar aquellos elementos favorables para el desarrollo sano de los niños y adolescentes atendidos. De tal manera que el trabajo en estas áreas se hace prioritario.

Por otro lado, la asociación establecida indica que sí existe relación entre resiliencia, autoestima y personalidad resistente, por lo que puede señalarse que los tres constructos se encuentran asociados entre sí. Estos resultados son consistentes con Wallace y Bisconti (2001), dado que la investigación en el área examina la personalidad resistente como un moderador de la resiliencia. Sin embargo, ésta es una asociación moderada por lo que se hace necesario seguir indagando acerca del sentido y la importancia de cada variable sobre la resiliencia en niños y adolescentes, así como la manera en que otras variables psicológicas mediadoras puedan estar involucradas y verificar su impacto en la resiliencia.

Considerando lo anterior, la comprensión de la relación entre estas variables: resiliencia, autoestima y personalidad resistente, es claramente compleja y más aún cuando se trata del estudio del maltrato infantil, desde su conceptualización hasta su medición y esto mismo abre la posibilidad de realizar mayor investigación al respecto.

Investigaciones futuras, deben de considerar estudios longitudinales para determinar más claramente la interacción de estas variables y proveer mayor información acerca de la personalidad de los individuos sobrevivientes al maltrato y abuso infantil.

Finalmente, se hace necesaria la promoción de la resiliencia en estos niños y adolescentes a fin de propiciar un desarrollo sano a través de intervenciones que los fortalezcan, puesto que se tiene la convicción de que es posible favorecer su resiliencia. 


\section{Referencias}

Affleck, G. \& Tennen, H. (1996). Construing benefits from adversity: Adaptational significance and dispositional underpinning. Journal of Personality, 64, 899-922.

Aracena, M., Castillo, R., Haz, A.M., Cumsille, F., Muñoz, S., Bustos, L. y Román, F. (2000). Resiliencia al maltrato físico infantil: variables que diferencian a los sujetos que maltratan y no maltratan físicamente a sus hijos en el presente y que tienen historia de maltrato físico en la infancia. Revista de Psicología de la Universidad de Chile, 9, 11-28.

Ammerman , R. T. \& Hersen, M. (1990). Research in child abuse and current status and an agenda for the future. En R.T. Ammerman y M. Hersen. (Eds.). Children at risk. An evaluation of factors contributing to child abuse and neglect (pp. 3-10). New York: Plenum Press.

Calhoun, L. G. \& Tedeschi, R. G. (1999). Facilitating Posttraumatic Growth: A Clinician's Guide. Mahwah, N.J.: Lawrence Erlbaum Associates Publishers.

Calhoun, L. G. \& Tedeschi, R.G. (2001) Posttraumatic growth: The positive lesson of loss. En Neimeyer, R.A. (Ed.). Meaning construction and the experience of loss. 157-172, Washington, D.C.: American Psychological Association.

Coopersmith, S. (1967). The antecedents of self-esteem. San Francisco: Freeman.

Davey, M., Goetter, D. \& Walters, H. L. (2003). Resilience processes in adolescents: personality profiles, self-worth and coping. Journal of Adolescent Research, 18(4), 347-362.

Dumont, M. \& Provost, M. A. (1999). Resilience in Adolescents: protective role of social support, coning strategies, self-esteem, and Social activities on experience on stress and depression. Journal of Youth and Adolescence, 28(3), 343-363.

Feinavver, L., Hiltl, G.H. \& Callahan, E. H. (2003). Hardiness as a moderator of shame associated with childhood sexual abuse. The American Journal of family therapy, 31, 65-78.

Frías, M., Castell, R. I. e Ibarra, S. P. (2000). Evaluación de un programa de atención a niños maltratados. Psicología y Salud, 10(1), 55-77.

González Arratia, L. F. N. I. (En prensa). Resiliencia y personalidad en niños y adolescentes. Cómo desarrollarse en tiempos de crisis. Universidad Autónoma del Estado de México.

González Arratia, L. F. N. I. (2007). Factores determinantes de la resiliencia en niños de la ciudad de Toluca. Tesis no publicada de Doctorado en Investigación Psicológica. Universidad Iberoamericana. Departamento de Psicología, Posgrado.

Ghorbani, N. W. (2005). Hardiness scales in Iranian managers: evidence of incremental validity in relationships with the five factor model and with organizational and psychological adjustment. Psychological Reports, Part 1, 96(3), 775-781.

Jiménez, A. G. (2008). Resiliencia y vejez. Madrid. Portal Mayores. Lecciones de Gerontología, XV. Recuperado el 5 de abril de 2011 de http://www.imsersomayores.csic.es

Kobasa, S. C. (1982). Commitment and coping in stress resistance among lawyers. Journal of Personality and Social Psychology, 42, 707-717.

Kobasa, S. C., Maddi, S. \& Kahn, S. (1993). Hardiness and health. A prospective study: clarification. Journal of Personality and Social Psychology, 42(1), 168-177.

Kobasa, S. C. (1979). Stressful life events, personality and health: An inquiry into hardiness. Journal of Personality and Social Psychology, 37(1), 1-11.
Lara, M. E., Martínez, C. y Pandolfi, M., Penroz, K. y Díaz, F. (2000). Resiliencia la esencia humana de la tranformación frente a la adversidad. CAD. "La esmeralda". Recuperado el 10 de diciembre de 2010 de http://www.udec.cl?clbustos/apsique/deli/-3k- .

Löesel, F., Bliesener, T. \& Kferl, P. (1989). On the Concept of Invulnerability: Evaluation and First Results of the Bielefeld Project. En M. Brambring, F. Löesel \& H. Skowronek (Eds.). Children at Risk: Assesment, Longitudinal Research and Intervention 186-219. (1989) New York: Walter de Gruyter.

Loredo, A. A. (1994). Maltrato al menor. México: Mc Graw Hill Interamericana.

Malinosky-Rummel, R. \& Hanse, D. (1993). Long-term consequences of childhood abuse. Psychological Bulletin, 114(1), 68-79.

Manciaux, M. (2003). La Resiliencia: resistir y rehacerse. Madrid: Gedisa.

Martínez, V. P. (2006). Significado psicológico de padre y madre en sujetos albergados en una casa hogar de la ciudad de Toluca. Tesis no publicada de licenciatura. Facultad de Ciencias de la Conducta. U.A.E.M.

Miguens, M. (2004). Gestalt y Resiliencia en la adversidad. Recuperado el 22 de mayo de 2011 de http://www.transpersonalpsycho.com.ar/ biblioteca/Resiliencia.htm

Moreno, B., Alonso, M. y Alvarez, E. (1997). Sentido de coherencia, personalidad resistente, autoestima y salud. Revista de Psicología de la Salud, 9(2), 115-137.

Reppucci, N. C., Britner, P. A. \& Woolard, J. L. (1997). Preventing child abuse and neglect through parent education. Baltimore, MD.: Paul $\mathrm{H}$. Brookes Publishing.

Rosemberg, M. (1965). Society and the adolescent self-image. Princeton. Princeton University Press. Traducido al castellano (1973). La imagen del adolescente y la sociedad. Buenos Aires: Paidós.

Trianes, T. V. y Gallardo, C. J. A. (1997). Influencia del sexo y la edad en las repercusiones psicológicas de los niños maltratados, físicamente. Psicothema. 9(3), 473-485.

Vera, B., Carbelo, B. y Vecina, M. (2006) La experiencia traumática desde la psicología positiva: resiliencia y crecimiento postraumático. $\mathrm{Pa}$ peles del Psicólogo, 27(1), 40-49.

Verduzco, A. I. M. A. (2004). Autoestima, Estrés y Afrontamiento desde el punto de vista del desarrollo. Tesis no publicada de Doctorado. Facultad de Psicología Universidad Nacional Autónoma de México.

Vinaccia, S., Quiceno, J. M. y Moreno, S. P. E. (2007). Resiliencia en adolescentes. Revista Colombiana de Psicología, 16, 139-146.

Wallace, K. A. \& Bisconti, L. T. (2001). The mediational effect of hardiness on social support and optimal outcomes in later life. Basic and applied social psychology, 23(4), 267-279.

Walsh, F. (2004). Resiliencia familiar. Buenos Aires: Amorrortu.

Werner, E. (1989). High-risk children in young adulthood: A longitudinal study from birth to 32 years. American Journal of Orthopsychiatry, 59(1), 72-81.

Widom, C. S. (1989). Child abuse, neglect and adult behavior: Criminality, violence and abuse. American Journal of Orthopsychiatry, 59, 355-367.

Williams, Z. A. (2001). Resiliency in Zimbabwean hildren impacted by HIVIAIDS. A clinical dissertation. Doctor of Psychology. University the California Scholl of Professional Psychology. Alameda. 